\title{
Flexible vacuum vessel bolometer camera design in ITER to adapt to the final position of the gaps between Blanket Modules
}

\author{
A. Pataki ${ }^{1}$, D. Hermann ${ }^{1}$, L.C. Ingesson ${ }^{3}$, H. Meister ${ }^{1}$, G. Nadasi ${ }^{2}$, F. Penzel ${ }^{1}$, R. Reichle ${ }^{4}$, \\ J. Sanchez ${ }^{5}$ U. Walach ${ }^{3}$, \\ ${ }^{1}$ Max-Planck-Institut für Plasmaphysik, Boltzmannstr. 2 D-85748 Garching, Germany \\ ${ }^{2}$ Wigner Research Centre for Plasmaphysics, Hungarian Academy of Sciences, Konkoly-Thege Miklos 29-33, H-1121 Budapest, Hungary \\ ${ }^{3}$ Fusion for Energy, c/ Josep Pla, $n^{\circ} 2,08019$ Barcelona, Spain \\ ${ }^{4}$ ITER Organization, Route de Vinon-sur-Verdon, CS 90 046, 13067 St. Paul Lez Durance Cedex, France \\ ${ }^{5}$ FIRCROFT, Lingley House, 120 Birchwood Point, Warrington, Cheshire WA3 7QH, United Kingdom
}

\begin{abstract}
Bolometer cameras in ITER will be mounted, among others, on the Vacuum Vessel (VV) wall behind Blanket Modules (BMs). For the first assembly phase the platform (Cable fixations and the lower part of the internal signal chain) of VV cameras has to be delivered to fix the cables and protect their termination. During First Plasma, the as-built magnetic axis and magnetic flux surface will be measured and the BMs fixation will be adjusted to align them to the shape of the magnetic flux surfaces. Adjustments of the BMs of up to $\pm 13,3 \mathrm{~mm}$ in toroidal and poloidal direction and up to $\pm 20,3 \mathrm{~mm}$ in radial direction are foreseen. Accordingly, VV cameras need adjustments, too, to assure a proper view of the plasma.

Calculations have been performed to define the impact of the possible BM movements onto the bolometer viewing cones. The toroidal and poloidal movements can be followed by shifting the collimator and the sensor along with the BMs. The radial movement of BMs was transferred to an additional poloidal shift of the sensor and collimator. The resulting concept for camera design with its complex space envelope will be presented. It provides an alignment flexibility of the cameras of $\pm 31 \mathrm{~mm}$ poloidally and $\pm 16 \mathrm{~mm}$ toroidally. Because of the short period between magnetic measurements and second assembly phase, the number of customizable parts is kept as low as possible.
\end{abstract}

Keywords: ITER, diagnostics, bolometer camera, plasma radiation, viewing cone

\section{Introduction}

The ITER Bolometer Diagnostic comprises a system of bolometer cameras located in two Upper Port Plugs, one Equatorial Port Plug, five divertor cassettes and 22 in-vessel positions in six different Vacuum Vessel (VV) sectors with a total of about 550 lines of sight (LOS) Ref [1].

With a largely constant efficiency from soft-X ray to infrared range, the diagnostic is the most important contributor to the measurement of the total radiated power for machine protection and its spatial distribution for basic control, advanced control and physics investigations.

Each VV camera consists of 5 LOSs. These LOSs together make up the viewing cone of the camera.

One of the most challenging tasks is to design the 22 in-vessel cameras and mount them in two steps to the VV wall.

\section{Vacuum vessel bolometer cameras}

VV cameras will be installed behind Blanket Modules (BMs) on the VV wall and will observe the plasma through the gaps between BMs. For providing better view to the plasma, most of the related BMs have a cut-out on the side, for the viewing cone of the camera. At the rear is another cut-out for the camera itself (Fig.1).

The cut-out for the viewing cone assures up to 30 $\mathrm{mm}$ wide clear view of the plasma between BMs.

During first plasma, the as-built magnetic axis will be measured and the BMs fixation will be adjusted to align the BMs to the shape of the resulting magnetic flux surfaces. Adjustments of the BMs of up to $\pm 13,3 \mathrm{~mm}$ in toroidal and poloidal direction and up to $\pm 20,3 \mathrm{~mm}$ in radial direction are foreseen Ref [2, 3]. The nominal gap between BMs is 14-20 $\mathrm{mm}$. It is assumed, that all BMs will move very similar to each other. Therefore, the relative distance between each other will not change.

The lower part of the camera, called platform will be attached to the VV wall using two bosses, i.e. rings with internal thread that are welded to the wall, during the first installation phase of ITER, in order to fix and protect the termination of mineral insulated cables (MIC). As the platform has been already installed, the final position of the BMs has to be taken into account in the design of the upper part of the camera. Some parts (as few as possible) of the bolometer upper assembly have to be customizable to be able to follow the BMs. 


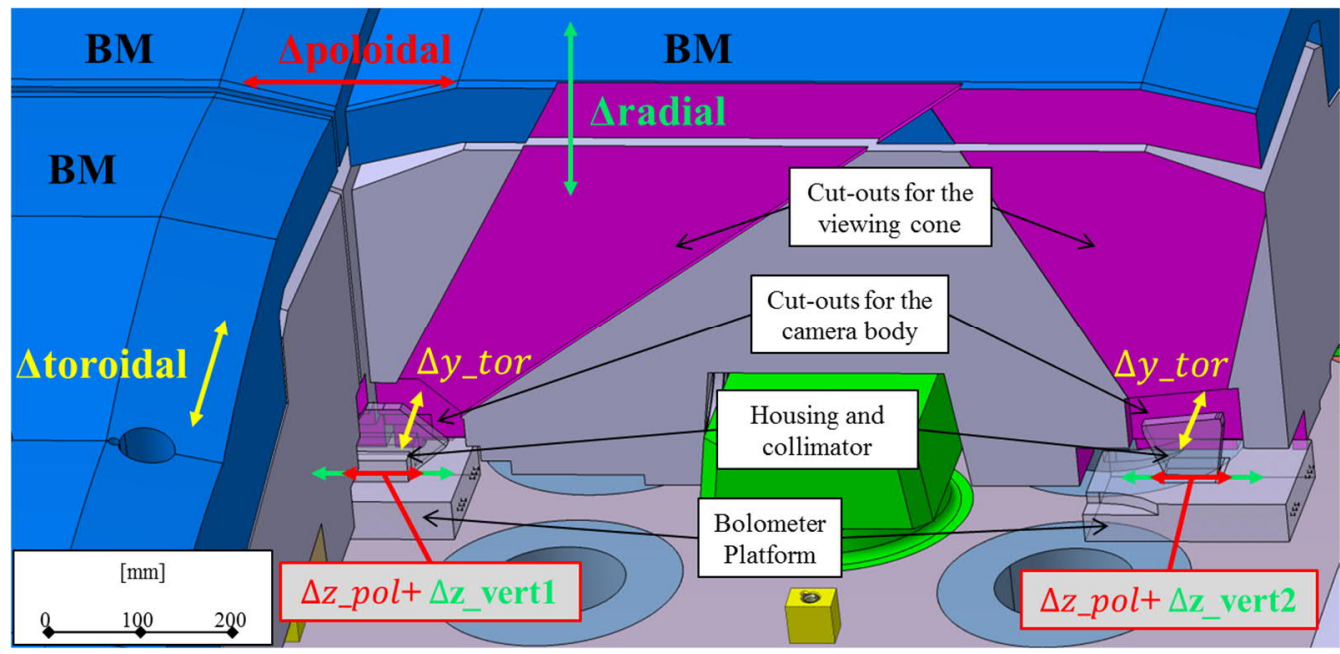

Fig. 1. Bolometer cameras behind BMs (blue and grey), and the dedicated cut-outs (pink)

\subsection{Impact of BM adjustment range on Bolometer camera design}

All components inside the VV will be installed with tolerances. This mounting tolerance can be added to the planned BM adjustments. The resulting values give the tolerances bolometer cameras have to adapt to and implement as an alignment flexibility. Alignment flexibility means the possibility of shifting the collimator and the sensor by customization of the upper part of the camera with respect to the platform. The situation is shown in Figs. 1 and 2. The pink areas show the cut-outs for both the viewing cone and the camera body.

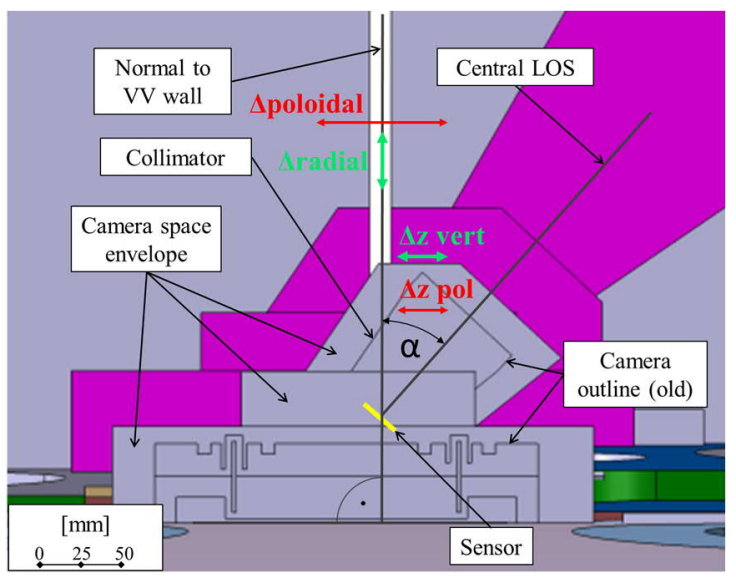

Fig. 2. Side view of bolometer camera and BM, defining the directions of the tolerances for calculating the alignment flexibility. Colours as in Fig. 1.

Calculation parameters are:

- $\quad \alpha$ - viewing angle, measured between the central LOS of the viewing cone and the normal to $\mathrm{VV}$ wall.

- $\quad$ poloidal - poloidal adjustment of BMs.
- $\quad$ radial - Radial adjustment of BMs, max \pm $20,3 \mathrm{~mm}$.

Toroidal and poloidal adjustments of the BMs can be followed by customizing the camera housing so that the collimator and the sensor shift along with the BM adjustments. The value of the shift ( $\Delta y_{-}$tor and $\Delta z \_$pol, Fig.1. $)$is $\pm 16 \mathrm{~mm}$ in both cases $( \pm 13,3 \mathrm{~mm}$ from the BM adjustments and $\pm 2 \mathrm{~mm}$ from the mounting tolerance of the bosses, rounded up to be on the conservative side). This shifting does not require any additional cut-outs for the camera body, because the relative position of the adjustable camera elements and the BMs remains the same.

However, the radial adjustment cannot be followed by a simple shift of collimator and sensor in radial direction. The sensor temperature has to be kept as low as possible for reasons of performance, so an elevation of the sensor and the sensor holder elements cannot be implemented: the increased thermal path would increase the sensor temperature. Therefore, the radial adjustment of BMs was transferred to an additional poloidal shift of the sensor and collimator to be able to keep the viewing cone aligned with the cut-out.

The value of the maximal possible shift has been calculated for all $22 \mathrm{VV}$ cameras, using the parameters shown in Fig. 2.

The radial adjustment Aradial of the BM is translated into a poloidal adjustment of the camera, $\Delta z \_v e r t$ according to the tangent of the angle $\alpha$ :

$$
\tan (\alpha)=\frac{\Delta z_{-} \text {vert }}{\Delta \text { radial }}
$$

The resulting poloidal shift values are summarized in Table 1 . The table also gives $\Delta z_{\text {_total, }}$, the sum of 
$\Delta z \_$vert and the maximal $\Delta z \_p o l=16 \mathrm{~mm}$, and $\Delta z \_$des. The latter is a weighted average of $\Delta z_{-}$vert and the maximum $\Delta z \_$pol according to

$$
\Delta z_{-} d e s=\sqrt{16^{2}+\left(\Delta z_{-} \text {vert }\right)^{2}}
$$

Table 1. Alignment flexibility calculations

\begin{tabular}{|c|c|c|c|c|}
\hline $\begin{array}{c}\text { Camera } \\
\text { name }\end{array}$ & $\begin{array}{c}\alpha \\
{[d e g]}\end{array}$ & $\begin{array}{c}\Delta z_{-} \text {vert } \\
{[\mathrm{mm}]}\end{array}$ & $\begin{array}{c}\Delta z \text { total } \\
{[\mathrm{mm}]}\end{array}$ & $\begin{array}{c}\Delta z \_d e s \\
{[\mathrm{~mm}]}\end{array}$ \\
\hline 1011 & 6 & 2,1 & 18,1 & 16,1 \\
\hline 1021 & 44 & 19,6 & 35,6 & 25,3 \\
\hline 1051 & 23 & 8,6 & 24,6 & 18,2 \\
\hline 1072 & 15 & 5,4 & 21,4 & 16,9 \\
\hline 1081 & 13 & 4,7 & 20,7 & 16,7 \\
\hline 1182 & 52 & 26 & 42 & 30,5 \\
\hline 1184 & 52 & 26 & 42 & 30,5 \\
\hline 3021 & 42 & 18,3 & 34,3 & 24,3 \\
\hline 3082 & 14,4 & 5,2 & 21,2 & 16,8 \\
\hline 3184 & 1 & 0,4 & 16.4 & 16,0 \\
\hline 4021 & 43 & 18,9 & 34,9 & 24,8 \\
\hline 4183 & 4 & 1,4 & 17,4 & 16,1 \\
\hline 6072 & 12 & 4,3 & 20,3 & 16,6 \\
\hline 6082 & 13,7 & 5 & 20,4 & 16,8 \\
\hline 6184 & 4 & 1,4 & 17,4 & 16,1 \\
\hline 8022 & 44 & 19,6 & 35,6 & 25,3 \\
\hline 8072 & 2 & 0,7 & 16,7 & 16,0 \\
\hline 8184 & 4 & 1,4 & 17,4 & 16,1 \\
\hline 9022 & 43 & 18,9 & 34,9 & 24,8 \\
\hline 9071 & 7 & 2,5 & 18,5 & 16,2 \\
\hline 9072 & 20 & 7,4 & 23,4 & 17,6 \\
\hline 9082 & 23 & 8,6 & 24,6 & 18,2 \\
\hline \multicolumn{2}{|c|}{ Maximum: } & 26 & 42 & 30,5 \\
\hline
\end{tabular}

Due to lack of space inside the camera, the alignment flexibility of the internal parts, - (Fig 4.) has to be limited to $30,5 \mathrm{~mm}$ (rounded up to $31 \mathrm{~mm}$ ) according to $\Delta z$ _des column.

This means that there is the possibility, that more flexibility is needed than the interior allows. In this case the sensor holder together with the cover and the collimator will be adjusted by $31 \mathrm{~mm}$, while the BM cut-outs will be shifted at maximum by an additional $11 \mathrm{~mm}$. This can occur for 7 cameras (where $\Delta z$ _total $>31 \mathrm{~mm}$ ). In all 7 cases only one LOS (6\% of the 110 LOSs) might be partially shadowed (looking partly through the dedicated cutout and partly through the nominal gap between BMs).

So the cameras shall be designed for a maximum of $\pm 31 \mathrm{~mm}$ poloidal and $\pm 16 \mathrm{~mm}$ toroidal alignment flexibility.

\subsection{VV bolometer camera design: platform}

The platform had to be designed to allow the necessary alignment flexibility, i.e. sufficiently large for subsequent customization of upper part of camera.
After several iterations, the design shown in Figures 3-5 has been developed.

The platform (Fig.3.) is bolted down to two bosses, which have been welded prior to the VV wall. Then the MICs will be fixed to the platform and electrically bonded to the platform printed circuit board (PCB). The connection pads (Fig 3. right) will be connected via electrical tracks to the female parts of the remote handling (RH) connectors. The concept of the RH connector is based on the solution from ITER in-vessel magnetics diagnostic. The assembly will receive a temporary cover to protect the internal elements from the loads during first plasma.

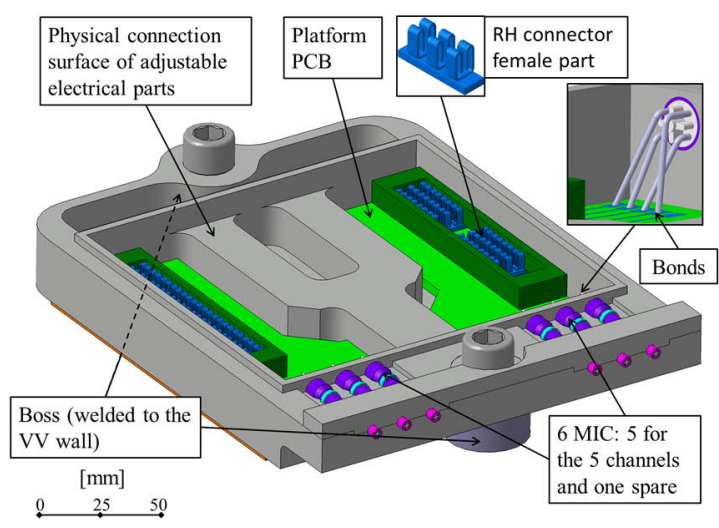

Fig. 3. Platform design

The as built position of the platform will be measured. This knowledge together with the calculated final position of the BMs will be the input for customizing the upper parts of the camera.

\subsection{VV bolometer camera design: adjustable electrical parts}

The adjustable electrical parts will be installed during the second assembly phase of ITER. The two sides of the RH connector will be spot-welded together to ensure a reliable electrical contact. The assembly is shown in Fig. 4.

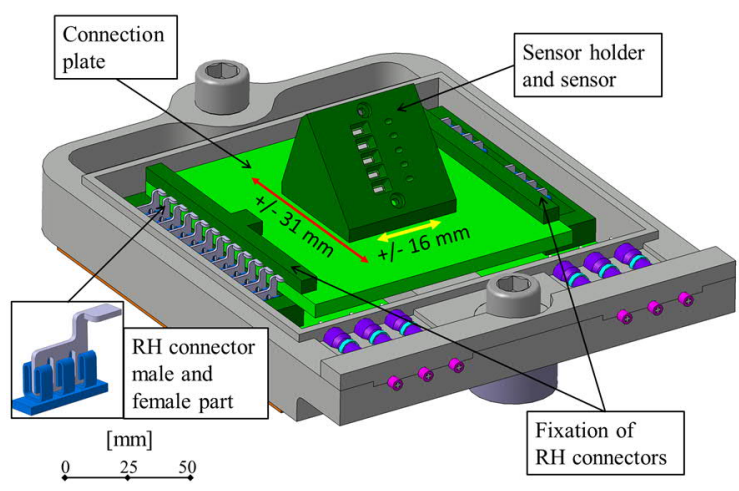

Fig. 4. Adjustable electrical parts 
Position of sensor holder on connection plate (CP) can vary to respect alignment flexibility as shown in the picture.

Because of the short period ( $\sim 6$ months) between magnetic measurements and the second assembly phase, the number of customizable parts should be kept as low as possible. In this case the fixation parts of the RH connectors, the sensor holder with the sensor and their electrical and physical connection can be manufactured in advance. The shape of the $\mathrm{CP}$ can also be prepared.

When the final sensor holder position is calculated, the tracks on the $\mathrm{CP}$ can be designed and manufactured. The male RH connectors and the sensor holder will be fixed to the CP. The electrical connection between the plate and the sensor holder will then be implemented.

\subsection{VV bolometer camera design: adjustable upper body}

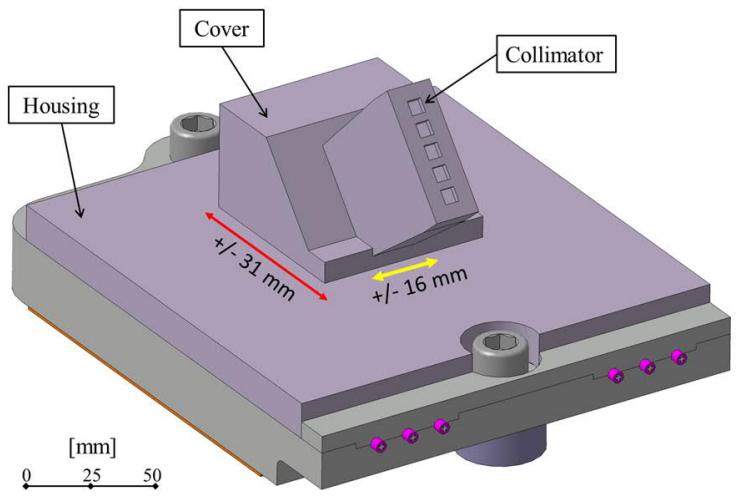

Fig. 5. Bolometer camera with installed upper parts

The upper assembly will be installed right after the adjustable electrical parts.

The collimator and the cover (Fig.5) can be fully manufactured and brazed together in advance. The shape of the housing can also be prepared and the cut-out for the cover and their fixation together will be done at a later stage.

This methodology assures that only a few components have to be designed (adjusted) and manufactured right before the second assembly phase of ITER.

\subsection{Space envelope of the VV camera}

A space envelope, - called Configuration Management Model (CMM), which is a theoretical volume in which the final camera has to fit in - needs to be established, including all tolerances and adjustment flexibility. The CMM is used to define the shape of the BM cut-outs (see in Fig. 1. and 2.).

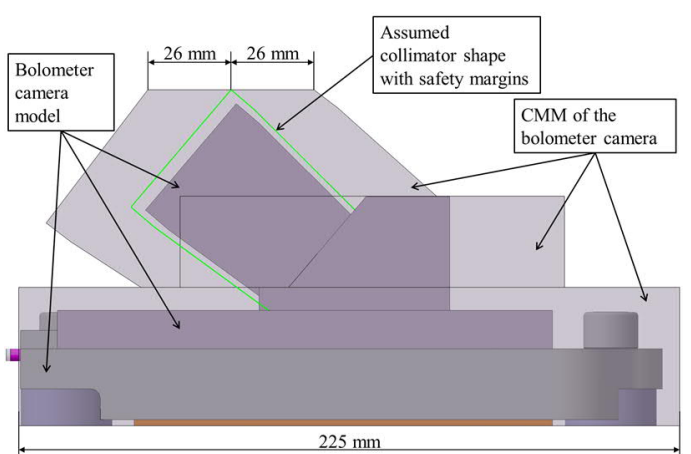

Fig. 6. VV bolometer camera and its space envelope

22 CMMs have been defined (one example is shown in Fig. 6). There are deviations between CMM (transparent) and detailed camera model (darker colors). The reason for this is the preliminary maturity level of the detailed model: Several components required for final design (e.g. captive bolts or RH pins) are not yet implemented. Furthermore some amount of space is foreseen for design the evolutions of the collimator.

The poloidal enlargement of the collimator and the cover for defining the space envelope is the outcome of implementing the alignment flexibility, (coming from the radial adjustment of the BMs). The values have been defined according to the $\Delta z_{-}$vert column of Table 1 . This prevents the possibility of physical clash between BM and camera and allows the bolometer designers to implement the $\Delta z_{-}$total alignment flexibility in case needed and in case the final design of the interior is compatible with this. At the moment the interior has been designed for \pm 31 $\mathrm{mm}$ flexibility in poloidal direction.

\section{Conclusions}

The final position of the BMs in ITER will deviate from their nominal position. Calculations have been performed to assess the necessary alignment flexibility of the VV bolometer cameras for adjusting the sensor and the collimator with the final position of the BMs and BM cut-outs.

The outcome of the calculation is a bolometer camera which can adapt a maximum of $\pm 16 \mathrm{~mm}$ toroidal- and $\pm 31 \mathrm{~mm}$ poloidal alignment flexibility. A design has been developed, which is able to handle this. Also, a set of CMMs has been prepared based on which the cut-outs on BMs have been defined to accommodate bolometer cameras.

\section{Acknowledgement}

This work was partly supported by Fusion for Energy under the Grant F4E-FPA-384-SG03. The views expressed in this publication are the sole 
responsibility of the authors and do not necessarily reflect the views of Fusion for Energy and the ITER Organization. Neither Fusion for Energy nor any person acting on behalf of Fusion for Energy is responsible for the use, which might be made of the information in this publication.

\section{References}

[1] H. Meister, et al. Current status of the design of the ITER bolometer diagnostic, Fusion Eng. Des., 120 (7) (2017), pp. 21-26

[2] L. Pierric, Assessment of clearances between VV and Blanket, 2016, Private Communication

[3] F.J. Fuentes, P. Leonard, Blanket Modules

Dimensional Variation Model 3.0 Compliance Status Assessment, 2014, Private Communication 